\title{
Nova Patrol at Abastumani
}

Givi N. Kimeridze, Omar M. Kurtanidze

Abastumani Astrophysical Observatory, 383762 Abastumani, Georgia

\section{The Telescope}

The Schmidt telescope (360-333-625 mm) of the Abastumani Astrophysical Observatory has been used for photographic observations for more than fifty years. It provides a field of $7.3(80 \mathrm{~mm})$ and a non-vignetted field of $4^{\circ}(44 \mathrm{~mm})$. A limiting magnitude of $16^{\mathrm{m}} 5 \mathrm{can}$ be reached in 7-8 min exposure on medium sensitivity astronomical films and $18^{\mathrm{m}}$ with fine grain emulsions.

Multicolour and polarization observations of a few hundred relatively bright galaxies were carried out by M. Vashakidze during the first two decades of the telescope operation. In the mid-1950s, he also discovered the strong polarization of the Crab nebula. Since 1961, systematic photographic observations are carried out in the framework of the International Cooperation Programme of Supernova Patrol in 24 fields. The telescope is also used to study the brightness variations of eruptive and exploding variable stars.

\section{The Patrol}

Systematic observations of novae began in the 1920 ies. Main data on the $\approx 300$ novae can be found in two catalogues (Kholopov et al. 1985, Duerbeck 1987). About one fourth of novae are not classified at all, and for many more the classification is not reliable.

According to Kholopov et al., it is possible to divide novae by their brightness variations into fast, slow, very slow and recurrent novae. Some authors are also pulling out the class of nova-like stars, which includes different, nonuniform groups of variables. Novae of different classes have different maximum brightnesses, but the differences are not yet well established (Hoffmeister et al. 1990).

A most critical parameter of novae is their flare frequency. Its determination is complicated because of several reasons: (a) there is no systematic wide-field nova patrol; (b) the discovery of novae in the galactic plane and especially in the galactic center direction is limited by interstellar absorption. It is possible to estimate the flare frequency by extrapolating the value for the same type of 
galaxy as our own. Arp (1956) and Sharov (1993) estimated the flare frequency of novae in M31 as 26 and 31 per year, respectively, while extrapolations of nova patrols in the Galaxy lead to the value 76 per year (Liller 1987).

Fast novae are uniformly distributed along galactic longitude, while slow novae show a tendency of concentration towards the direction of the Galactic center. This fact probably indicates that there are two types of novae in galaxies like our (Duerbeck 1990), while it is not confirmed for M31 (Sharov 1993). Sharov also indicated the expediency of nova patrols in M31 and the Galaxy.

There are also much problems connected with recurrent novae. It is not yet known whether all novae are recurrent. Kaluzny and Glebowski give a list of potential recurrent novae for patrol observations. The selected objects have generally a low maximum brightness which is statistically correlated with short intervals between nova outbursts. Only one object from this list - V1017 Sgr appears as recurrent in nova catalogues.

It should be emphasized that there are connections between novae and novalike objects (dwarf novae, symbiotic stars, polars) and planetary nebulae. Only a few polars are known up to now, so it is desirable to carry out a nova patrol also in this respect.

We plan to initiate a nova patrol at Abastumani, using the Schmidt telescope. The 59 selected fields fully cover the following three strips: $\ell=0^{\circ}-30^{\circ},|b|<$ $12{ }^{\circ} 5, \ell=30^{\circ}-60^{\circ},|b|<7^{\circ} .5$, and $\ell=60^{\circ}-90^{\circ},|b|<5^{\circ}$. We plan to use A660N photographic films with maximum sensitivity at $\mathrm{H} \alpha$ with wavelength $6000-6700 \AA$ (Novae have strong $\mathrm{H} \alpha$ emission during outburst) and avoiding blue field stars and blue background. With 5 min exposures, the whole patrol cycle may be completed even during two summer nights.

This material will also be used for the identification and subsequent investigation of the variability of late type stars ( $\mathrm{M}$ and $\mathrm{C}$ stars), detected in a low dispersion spectral survey (Kurtanidze \& Nikolashvili 1994). Finally, we like to note that any suggestion for cooperation in Nova Patrol Programme is very welcome.

Acknowledgements: One of the autors (O.M.K.) is grateful to the SOC of the conference for financial support to attend.

\section{References}

Arp, H.C. 1956, Astr.J., 61, 15

Duerbeck, H.W. 1987, Space Science Reviews, 4

Duerbeck, H.W. 1990, in Physics of Classical Novae, IAU Coll. no.122, ed. A. Cassatella \& R. Viotti, Springer, Berlin, p. 34

Hoffmeister, C. et al. 1990, Variable Stars, Nauka, Moscow

Kaluzny, J., Chlebowski, T. Preprint CfA no.2667

Kholopov, P.N. et al. 1985, General Catalogue of Variable Stars, Nauka, Moscow

Kurtanidze O.M., Nikolashvili M.G. 1994, in Astronomy from Wide-Field Imaging, IAU Symp. 161, p. 467

Liller, W. 1987, APSS 131, 449

Sharov, A.S. 1993, Pisma Astr. Zhurn. 19, 18 